\title{
Learner Modelling in Exploratory Learning for Mathematical Generalisation
}

\author{
Mihaela Cocea \\ London Knowledge Lab, Birkbeck College \\ 23-29 Emerald St, WC1N 3QS London (UK) \\ mihaela@dcs.bbk.ac.uk
}

\begin{abstract}
Exploratory learning supports creative thinking, allowing learners to control their own learning process, whilst it provides them with help and guidance when necessary. This pedagogical approach emphasises learners' active involvement in authentic activities/tasks that simulate real world processes and has been applied to several domains. In this paper we propose a framework for learner modelling that reflects the incremental nature of knowledge construction as learners are engaged in learning mathematical generalisation. We also describe how such a model can potentially support feedback generation.
\end{abstract}

Keywords: learner modelling, exploratory learning, feedback generation, mathematical generalisation.

\section{Introduction}

Constructivism [11] sees learning as an active, constructive process in which knowledge is built and structured gradually. Exploratory/discovery learning supports this view of learning and has been argued to be particularly beneficial [2] in terms of providing opportunities for acquiring deep conceptual and structural knowledge. However pure discovery learning without any guidance and support is hardly beneficial [4]. The main challenge with this approach is to balance freedom with control: learners should be given enough freedom so that they can actively engage in constructing models and they should be offered enough guidance in order to assure that their constructions lead to useful knowledge [7].

Besides the clear and well-acknowledged challenge of balancing freedom with guidance, there are other issues that make the process of learner modelling in Exploratory Learning Environments (ELEs) demanding:

- What to model? Usually learner models relate to knowledge or skills. In the context of exploratory learning, the knowledge results from constructionist processes and there is a clearer indication of this knowledge at the end of these processes. Nevertheless, support is required both during knowledge construction and at the end of certain processing stages. Thus, a key question is what to model so that support can be provided during and at the end of knowledge construction.

- Value of correct vs. incorrect actions. In most e-Learning systems, feedback is related to correctness or incorrectness of answers/actions, while in ELEs learner's explorations are difficult to categorise into correct or incorrect. Moreover, even if such a classification would be possible, incorrect actions may be more valuable for learning than correct ones. Actually, one of the advantages of ELEs is that learners 
are given the opportunity to realise their own mistakes and learn from them; thus, rather then pointing out possible mistakes, the system should provide learners with feedback that would encourage reflection on their actions and help them realise that their knowledge construction is not entirely correct.

- Relation between abstract knowledge and forms of (re)presentation in the system. ELEs have different ways of (re)presenting and exploring models that should gradually help the learner build abstract knowledge. Each part of the model and each type of exploration (e.g. changing parameters, creating new models, testing models etc.) contributes to this process. Identification of relevant abstract knowledge is needed as well as its representation in the learner model.

- Identification of underlying strategies from actions or sequences of actions. Sometimes is neither realistic nor feasible to include all possible outcomes (correct or incorrect) and ways to achieve them when modelling an extensive knowledge domain. Thus, a different approach to what is included in the knowledge structure is required; rather than storing complete information about a task or expert knowledge, key information with informative educational value could be stored such as strategies for approaching the (sub)task and landmarks indicating a particular strategy or (lack of) knowledge about a particular aspect. The challenge is how to find this information and how to represent it in the knowledge structure.

Given the abovementioned challenges, a classical approach to learner modelling based on concepts would not fit the purposes of ELEs. The classic approach involves a particular scenario: learners are required to study materials about a concept and then their knowledge level is assessed through testing. On the contrary, ELEs involve knowledge discovery by means of constructive activities and the emphasis is on the process rather that the knowledge itself and thus, the learner modelling process should reflect this way of learning. The nature of this process places the focus on the interactions of the learner with the system rather than on their answers to tests. Thus, analysing interactions during knowledge construction and extracting relevant information is an essential part of the learner modelling process that together with knowledge about student's learning processes inferred from their models and their learning progression can play an important role in generating feedback and support.

In this paper, we propose a framework for learner modelling in ELEs that follows the principles of constructivism and supports provision of feedback in order to guide the learner towards useful and sound knowledge construction. The following section gives a brief overview of previous research in ELEs and introduces our research questions. In Section 3, our framework is presented together with the methodology and one example. Section 4 presents the expected contributions of our research.

\section{Background and Research Questions}

We briefly present here three approaches to support exploratory learning: (a) heuristics were used by [10] to guide the learning process for a physics domain; (b) Bayesian networks were used by [1] for the mathematical functions domain; (c) a neuro-fuzzy approach was proposed by [9] for student diagnosis for a physics domain.

The idea of intelligent support is tackled in the first approach using induction and deduction, whilst templates are used to generate feedback; no learner model is used. 
The second approach addresses "effective exploration" [1], but uses "standard" student modelling in the sense that essential cases for the problems to be explored are used as the equivalent of concepts in classical overlay models. Two of the challenges previously mentioned, i.e. what to model and the difficulty of determining the (in)correctness of an action, were also addressed. The third approach uses knowledge of experts in teaching physics encoded in the form of fuzzy sets and rules and applies training from practical examples when teachers' knowledge is not accurate or welldefined; the purpose was student diagnosis and no feedback is provided.

In contrast to previous attempts, here we advocate an approach that extends user modelling in ELEs by reflecting and supporting the constructionist learning process. Since the focus is on the process, interaction analysis [8] plays an essential part in learner modelling. Typically it starts with filtering raw data in order to extract some indicators related to the quality of the learning process. These indicators can be used for several purposes; in our case, the main purpose is the regulation of the learning process through feedback, while a secondary purpose is to inform teachers about students' learning process and progression. Thus, the research questions addressed in our research are the following: (a) What interactions are relevant and how can they be extracted them from the flow of raw data and transformed into indicators? (b) What should be stored in the learner model in order to represent the evolution of the learner's constructionist models and their corresponding cognitive processes? (c) How should the learner model be updated in order to reflect both the current knowledge and the evolution of knowledge? (d) Using the learner model, how can personalised feedback be provided to support the constructionist process and inform the teacher?

\section{Proposed framework and methodology}

In our framework the ELE includes two components (see Fig. 1): a domain and a task model. The domain model includes high level learning outcomes related to the domain and considers that each learning outcome can be achieved by exploring several tasks. The task model includes different types of information: (a) strategies of approaching the task which could be correct, incorrect or partially correct; (b) outcomes of the exploratory process and solutions to specific questions associated with each (sub)task; (c) landmarks, i.e. relevant aspects or critical events occurring during the exploratory process; (d) context, i.e. reference to this particular task.

In our approach, the structure of the learner model and the updating process follow the model of human memory often used in user modelling (e.g. [5]), and includes two components: a short-term model (STM) and a long-term model (LTM). The STM includes recent actions of the learner. The LTM contains information about the domain and the task and thus has two parts: the Task LTM that has the same structure as the task model, and the Domain LTM, which is an overlay model of the domain and maintains the knowledge of the learning outcomes associated with the learning process as inferred from the learner's constructions.

The learner model update and feedback generation are illustrated in Fig. 1. Recent actions of the learner (raw data) are stored in the STM. They are pre-processed and the transformed data are matched to cases from the Task Model; any identified strategies together with landmarks (if any), outcomes and context are stored or 
updated in the Task LTM. Based on Task LTM, Task Model and Domain LTM feedback is generated. Finally, the degree of meeting the learning outcome that was explored through the (sub)task is updated in the Domain LTM. Thus, the modelling process reflects the constructionist approach of incremental knowledge acquisition.

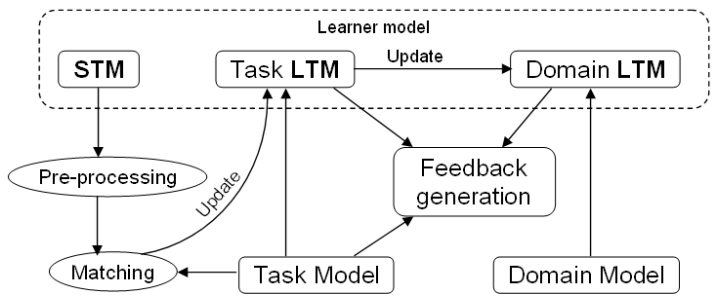

Fig. 1. Learner modelling process.

The learner modelling process supports two types of feedback: during the exploration process and at the end of certain processing stages. The first one aims to guide the learner in gradually constructing the knowledge, while the second one is more related to outcomes of the exploration and specific solutions.

Our framework will be validated by incorporating it into an ELE for mathematical generalisation developed in the context of MiGen project ${ }^{1}$ and testing in classrooms. To illustrate our approach we use an example from this domain and a task called 'pond tiling', which is common in the English school curriculum and expects learners to produce a general expression for finding out how many tiles are required for surrounding any rectangular pond. The high level learning outcome in the Domain Model is the students' ability to perform structural reasoning. In order to achieve this, subtasks can be explored, e.g. construct a pond of fixed dimensions, surround it with tiles and determine how many are required; generalise the structure using variables.

The Task Model (Fig. 2) could contain: (a) strategies, e.g. thinking in terms of width and height, thinking in terms of areas; (b) landmarks, e.g. creating a rectangle that has the height and width of the pond incremented by two as an indication of the 'areas strategy'; (c) outcomes (e.g. model built, numerical answer for a particular pond) and solution, i.e. a general algebraic expression (e.g. 'areas strategy': $($ width +2$) *($ height +2$)-$ width * height $)$; (d) context, i.e. reference to the task.

\begin{tabular}{|c|}
\hline Outcomes \\
\hline $\begin{array}{l}\text { Model: constants/ } \\
\text { variables/ } \\
\text { dependency }\end{array}$ \\
\hline $\begin{array}{l}20 \text { tiles needed } \\
\text { for a } 5 \text { by } 3 \text { pond }\end{array}$ \\
\hline
\end{tabular}

\begin{tabular}{|c|c|c|}
\hline Landmarks & Strategies & Solutions \\
\hline Rows/columns of & Width and height & $(\mathrm{w}+2) * 2+\mathrm{h} * 2$ \\
\hline & Areas & $\begin{array}{c}(\mathrm{w}+2)+2+\mathrm{h}^{*} 2 \\
2 \mathrm{w}+2 \mathrm{~h}+4\end{array}$ \\
\hline $\begin{array}{l}\text { Constants/variables } \\
\text { dependency }\end{array}$ & & $\frac{(\mathrm{w}+1) * 2+(\mathrm{h}+1) * 2}{(\mathbf{w}+\mathbf{2}) *(\mathbf{h}+\mathbf{2})-\mathbf{w}^{*} \mathbf{h}}$ \\
\hline
\end{tabular}

Fig. 2. Partial task model (slots connected by solid lines correspond to the example in the text).

During the task, the actions of the learner are stored in the STM and preprocessed. This process aims to transform the raw data into intermediate level data that will be used to identify (match) the relevant strategies, landmarks, outcomes and solutions for a learner in the current task or subtask. Knowledge of the domain and teachers' expertise together with findings from pilot studies will be used to derive

\footnotetext{
${ }^{1}$ The MiGen project is funded by ESRC/EPSRC; project website: http://www.lk1.ac.uk/cms/index.php? option $=$ com $\quad$ content\&task $=$ view\&id $=193 \&$ Itemid $=91$
} 
these aspects for every (sub)task and define a 'light-weight' model for mathematical generalisation. For pre-processing, a technique similar to episodes identification and association [6] can be used and comparisons will be made using fuzzy similarity measures. After matching, the Task LTM is updated. At the end of the "generalise the structure with variables" subtask, the knowledge associated with variables manipulation, which is considered an important step in the process of developing mathematical reasoning and generalisation ability, is updated in the Domain LTM.

During the (sub)task, feedback is provided based on the Task Model, Task LTM and Domain LTM; e.g. if the learner has surrounded the pond following a strategy that does not generalise well, the feedback can suggest resizing of the pond, which would result in "messing up" [3] the model, and encourage the learner to reflect on what is missing in order to make the solution general.

\section{Concluding remarks and contribution}

Exploratory learning operates on the principle that knowledge is built gradually as a result of active participation in learning. In this context, we proposed a framework for user modelling and briefly described how the model can be used for feedback generation in mathematical generalisation. The expected contributions of this research are: (a) a novel framework for learner modelling that reflects the constructionist learning approach; (b) a mechanism for updating such a model and (c) usage of the learner model for personalised feedback in an ELE and for informing teachers.

\section{References}

1. Bunt, A., Conati, C.: Probabilistic Student Modeling to Improve Exploratory Behaviour. Journal of User Modeling and User-Adapted Interaction. 13(3), 269-309 (2003)

2. de Jong, T., van Joolingen, W.R.: Scientific discovery learning with computer simulations of conceptual domains. Review of Educational Research, 68, 179-202 (1998)

3. Healy L., Hoelzl R., Hoyles C., Noss, R.: Messing Up. Micromath, 10, (1) 14-16 (1994)

4. Kirschner, P. A., Sweller, J., Clark, R. E.: Why minimal guidance during instruction does not work: An analysis of the failure of constructivist, discovery, problem-based experiential and inquiry-based teaching. Educational Psychologist, 41(2), 75-86 (2006)

5. Li, L., Yang, Z., Wang, B., Kitsuregawa, M.: Dynamic Adaptation Strategies for LongTerm and Short-Term User Profile to Personalize Search. APWeb/WAIM, 228-240 (2007)

6. Liu, J., Wong, C.K., Hui, K.K.: An Adaptive User Interface Based on Personalized Learning, IEEE Intelligent Systems, Vol.18, No 2, 52-57 (2003)

7. Mayer, R.: Should There Be a Three-Strikes Rule Against Pure Discovery Learning? The Case for Guided Methods of Instruction. American Psychologist, 59 (1), 14-19 (2004)

8. Papanikolaou, K., Grigoriadou, M.: Modelling and Externalising Learners' Interaction Behaviour. In Proceedings of the LeMoRe05 workshop, AIED2005, 52-61 (2005)

9. Stathacopoulou, R., Magoulas, G.D., Grigoriadou, M., Samarakou, M.: Neuro-fuzzy knowledge processing in intelligent learning environments for improved student diagnosis, Information Sciences, 170, 273-307 (2005)

10. Veermans, K.H.: Intelligent support for discovery learning, $\mathrm{PhD}$ thesis, University of Twente (2003)

11. Vygotsky, L.S.: Mind and society: The development of higher mental processes. Cambridge, MA: Harvard University Press (1978) 